\title{
De novo Atypical Chromosome Translocation 46, XY, t(4;13)(q12;p12)dn in Prenatal Diagnosis
}

Chetta $\mathbf{M}^{*}$, Di-Matteo L, Russo M, Sodano E, Festa M and De-Feo G

Medicina Futura, Laboratory of Medical Genetics, Italy

\begin{abstract}
Apparently balanced reciprocal translocations are a common type of chromosome rearrangements with an estimate incidence range from about 1 in 500 to 1 in 625 human newborns. Rearrangements were found both in clinically unaffected individuals and patients with phenotypic abnormalities. Most are inherited, but approximately one in five are de novo events and introduce a risk of abnormal phenotype in $6.1 \%$ of prenatal genetic counseling.
\end{abstract}

Keywords: Chromosome; Prenatal diagnosis; Genetic material

\section{Introduction}

In the present case, we reported the analysis results of the proband for prenatal diagnosis using G-banded karyotype, C-banding, nuclear organizer regions (Ag-NOR) staining and whole genome array methodology $(\mathrm{CGH})$. We documented a de novo atypical balanced $46, \mathrm{XY}, \mathrm{t}(4 ; 13)(\mathrm{q} 12 ; \mathrm{p} 12) \mathrm{dn}$ with an unusual length of chromosome 13 (chr13) satellite stalk [1-5]. Initial chromosome analysis from amniotic fluid cultures samples were performed by standard G-banding of metaphase chromosomes. The karyotype analysis is extended on both parents culturing peripheral blood mononuclear cells in order to elucidate the chromosome rearrangements.

Karyotypes of proband and parents were established according to the International System for Human Cytogenetic Nomenclature (ISCN 2013) [6]. In addition, the proband rearrangement was analyzed using, C-banding, silver staining (Ag-NOR) for nucleolar organizing regions to highlight the unusual length of chr13 satellite stalks. Finally, the Cytogenetic analysis was completed using whole genome array CGH methodology.

\section{Chromosome preparation}

Karyotyping was executed on metaphase chromosome preparations from amniotic fluid obtained via amniocentesis (proband) and peripheral blood lymphocytes (parents) using standard G-banded metaphases (400-band level for proband and 550 band resolution for parents) by modifications of the trypsin (GTL) and Giemsa/Leishman staining following standard procedure. Chromosome analysis was accomplished twice on amniotic fluid collected on two separated occasions following the European Cytogenetic guideline $[7,8]$. Cytogenetic analysis was thorough on amniotic fluid by C-banding and Ag-NOR staining following standard methods. G-banded, AgNORstained and C-banding were examined under a light microscope attached to a computerized analysis system to capture grayscale. "tiff images (Zeiss Axio Imager.M2 and digital camera Cool Cube 1 MetaSystems).

\section{Array data and confirmatory analysis}

Array data set was analyzed using the Bluefuse Multi software (BlueGnome Ltd. Cambridge, UK), and the reporting threshold was set at $200 \mathrm{~kb}$. All called imbalances detected and copy number changes were compared to known aberrations listed in public available databases ENSEMBL (Ensembl: http://www.ensembl.org), DECIPHER (http:// decipher.sanger.ac.uk) and the Database of Genomic Variants (DGV, http://projects.tcag.ca/variation/) - using NCBI136/hg18 UCSC or
GRCh37/hg19 assemblies and were further aligned with the in-house database

\section{Case Report}

The proband was a female without detectable fetal morphostructural evidences or functional alterations after routine ultrasound screening. The age of mother and father are respectively 38 and 29 years old without apparent phenotypic abnormalities. There was no documented family history of known genetic disorder and the parents were in a non-consanguineous marriage. Parents already had two children of 18-month-old and 3-year-old, respectively, without evidence of genetic disorder. The cytogenetic study using G-banded karyotype in proband revealed an atypical translocation $46, \mathrm{XY}, \mathrm{t}(4 ; 13)(\mathrm{q} 12 ; \mathrm{p} 12) \mathrm{dn}$ with a peculiar length of satellite stalks of chr13. This unusual length of satellite stalks was highlighted using Ag-NOR staining. As shown by AgNOR staining the satellites of chr13 range in size from double to triple (panel. F). The abnormal length of satellite stalks and the consequent distance between the boundaries of the chr13 and chr14 makes the cytogenetic investigation extremely complicated. After obtaining informed consent, the cytogenetic analysis was extended to the parents. Parental origins of the translocation were excluded analyzing karyotyping of both parents at a minimum resolution of 550 band following the European Cytogenetic guideline and defining as de novo, the chromosome rearrangements identified. Finally, using array CGH the absence of genetic material lack in proband was confirmed and the non-appearance alterations in parents, corroborating the evidence of de novo balanced chromosomal translocation. However, CGH array investigation showed the presence of approximately 1.7 $\mathrm{Mb}$ paternal duplication which includes glutamate ionotropic receptor kainate type subunit 2 gene (GRIK2) on chr 6 located onto region 6q16.3 (102,337,371-104,071,324). A defect GRIK2 gene was associated with autosomal recessive mental retardation while there are no clinical evidences attributable to gene duplications (Figure 1).

*Corresponding author: Dr. Massimiliano Chetta, Medicina Futura, Laboratory of Medical Genetics, Italy, Tel: +39 089-96 504; E-mail: mchetta@unisa.it

Received October 24, 2018; Accepted November 05, 2018; Published November 08, 2018

Citation: Chetta M, Di-Matteo L, Russo M, Sodano E, Festa M, et al. (2018) De novo Atypical Chromosome Translocation 46, XY, t(4;13)(q12;p12)dn in Prenatal Diagnosis. J Mol Genet Med 12: 374 doi:10.4172/1747-0862.1000374

Copyright: $@ 2018$ Chetta M, et al. This is an open-access article distributed under the terms of the Creative Commons Attribution License, which permits unrestricted use, distribution, and reproduction in any medium, provided the original author and source are credited 


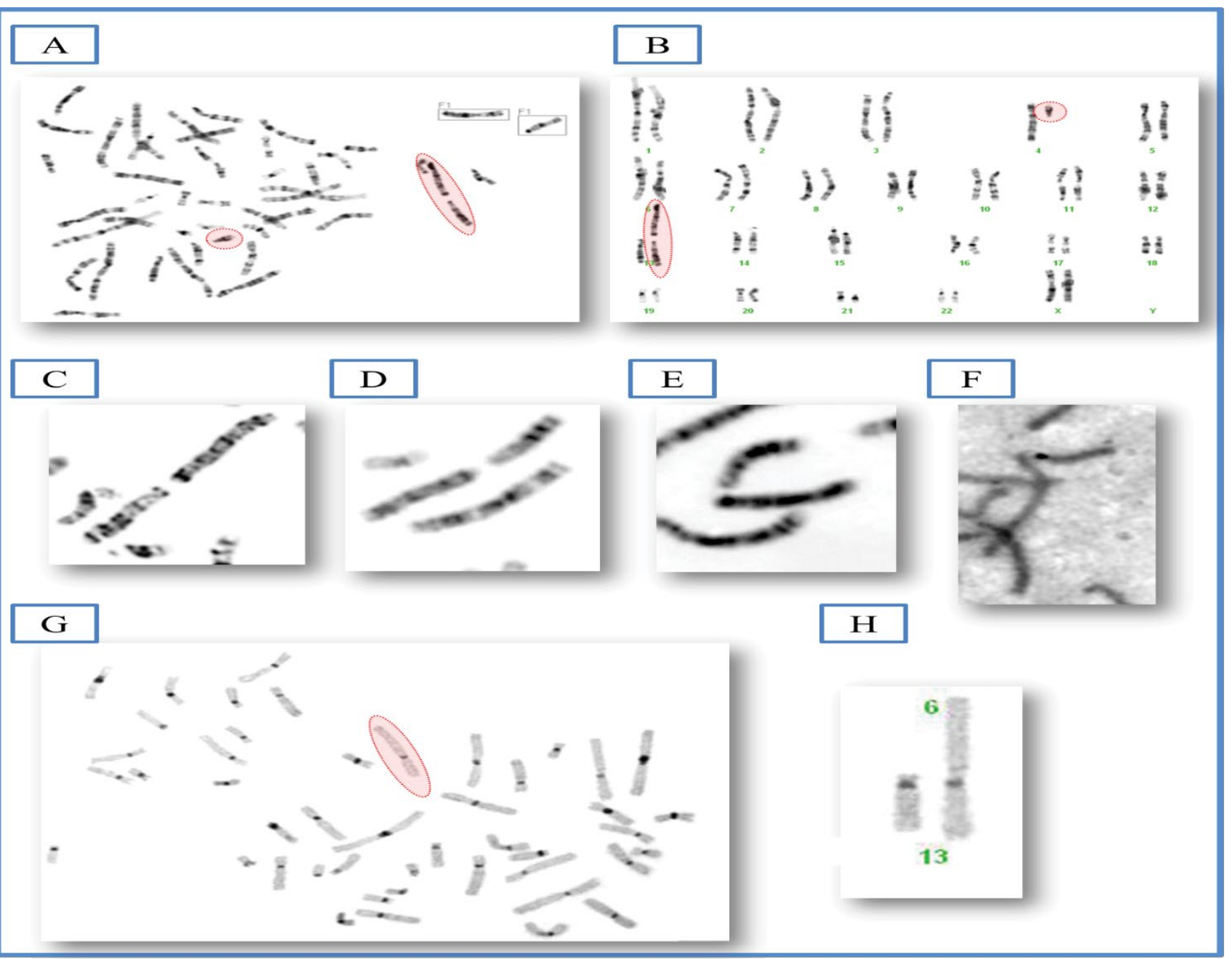

Figure 1: GTG-banding karyotype of amniotic fluid at the 400-band level. The translocation (4;13)(q12;p12)dn are highlighted by red circle (Panel A). Full proband karyogram (Panel B). Detail of translocation in three different metaphases where is clearly visible the peculiar length of satellite stalks of chromosome 13 (Panels C, D, E). Detail of translocation using Ag-NOR (Panel F). Proband metaphase and detail of translocation (4;13)(q12;p12)dn using C-banding (Panel G, H).

\section{Discussion}

Although the specific translocation $46, \mathrm{XY}, \mathrm{t}(4 ; 13)(\mathrm{q} 12 ; \mathrm{p} 12) \mathrm{dn}$ was not described previously there are some indications concerning the region $4 \mathrm{q} 12$ and $13 \mathrm{p} 12$. In particular the region $4 \mathrm{q} 12$ was associated to different kind of tumors. In literature, are reported deletions, duplications and inversions involving the $4 \mathrm{q} 12$ region and other portions of the 13 chromosomes (13q14, 13q22, 13q33). In these cases, the patients are affected by lymphoid neoplasms, Hypereosinophilic syndrome (HES), breast cancer and Central nervous system (CNS) tumors. The region $13 \mathrm{p} 12$, in the case of deletion or duplication inversion, was associated to prostate cancer [9]. Currently karyotype analysis is the most widespread routine genetic test in the world and continues to offer always new information. Autosomal reciprocal balanced translocations occur in approximately $0.1 \%$ of newborns and the estimated frequency of de novo balanced rearrangements in the general population from prenatal diagnosis is $0.0283 \%$ [10-12].

\section{Conclusion}

In our case, results should be interpreted carefulness because we have not information about a possible role of this translocation. We do not exclude the possible transposition of genetic material in active chromatin region, resulting in altered gene expression related to position effect, or presence of distal regulatory i.e., enhancer or silencer elements. Our case should require a cautious proband's follow up to observe possible abnormal phenotype and understand potential molecular features in order to classify specific disease by mapping possible gene breakpoints or position effect.

\section{References}

1. Jacobs PA, Browne C, Gregson N, Joyce C, White H (1992) Estimates of the frequency of chromosome abnormalities detectable in unselected newborns using moderate levels of banding. J Med Genet 29: 103-108.

2. Warburton D (1991) De novo balanced chromosome rearrangements and extra marker chromosomes identified at prenatal diagnosis: Clinical significance and distribution of breakpoints. Am J Hum Genet 49: 995-1013.

3. Howe B, Umrigar A, Tsien F (2014) Chromosome preparation from cultured cells. J Vis Exp 83: e50203.

4. Gill BS, Kimber G (1974) The Giemsa C-banded karyotype of rye. Proc Nat Acad Sci USA 71: 1247-1249. 
Citation: Chetta M, Di-Matteo L, Russo M, Sodano E, Festa M, et al. (2018) De novo Atypical Chromosome Translocation 46, XY, t(4;13)(q12;p12)dn in Prenatal Diagnosis. J Mol Genet Med 12: 374 doi:10.4172/1747-0862.1000374

Page 3 of 3

5. Goodpasture C, Bloom SE (1975) Visualization of nucleolar organizer region in mammalian chromosomes using silver staining. Chromosoma 53: 37-50.

6. Simons A, Shaffer LG, Hastings RJ (2013) Cytogenetic nomenclature: Changes in the ISCN 2013 Compared to the 2009 Edition. Cytogenet Genome Res 141: 1-6.

7. Barch M, Kaback M, Spurbeck J (1997) AGT Cytogenetics laboratory technical manual. Lippincott Williams and Wilkins, USA.

8. Mikhail FM, Watson MS (2014) Quality assurance and quality control in clinical cytogenetics. Curr Protoc Hum Genet 82: 8.2.1-8.2.10.

9. Fernandez BA, Siegel-Bartelt J, Herbrick JA, Teshima I, Scherer SW (2005)
Holoprosencephaly and cleidocranial dysplasia in a patient due to two positioneffect mutations: A case report and review of the literature. Clin Genet 68: 349-359.

10. Gardner RJM, Sutherland GR, Shaffer LG (2011) chromosome abnormalities detected at prenatal diagnosis. In: Chromosome abnormalities and counselling. (4th edn). Oxford University Press, USA.

11. Ogilvie CM, Scriven PN (2002) Meiotic outcomes in reciprocal translocation carriers ascertained in 3-day human embryos. Eur J Hum Genet 10: 801-806.

12. Oliver-Bonet M (2002) Aneuploid and unbalanced sperm in two translocation carriers: evaluation of the genetic risk. Mol Hum Reprod 8: 958-963. 